\title{
A Rare Cause of Intestinal Obstruction during Pregnancy: The Volvulus of the Small Intestine about a Case
}

\author{
Roufaida Ben Janan*, Yassir El Oukli, Siham Badaa, Najia Zeraidi, Abdelaziz Baidada
}

Service of Gynecology-Obstetrics and Endoscopy M1, maternity suissi Rabat

DOI: $\underline{10.36347 / \mathrm{sjmcr} .2020 . \mathrm{v} 08 \mathrm{i} 01.010}$

| Received: 04.01.2020 | Accepted: 11.01.2020 | Published: 16.01.2020

*Corresponding author: Roufaida Ben Janan

Abstract

Case Report

Volvulus of the small intestine is a rare complication of pregnancy, difficult and late diagnosis at the stage of complication since the clinic is not very specific, often camouflaged by a symptomatology that can be related to pregnancy. We report the case of a 38-year-old pregnant woman at 18 weeks of amenorrhea in a bowel obstruction chart with threat of abortion. Radiological exploration had found mechanical bowel obstruction with signs of digestive distress. Surgical exploration found a small intestine volvulus with necrosis requiring resection. In the light of this observation, the diagnostic and therapeutic modalities of this rare association will be discussed.

Keywords: pregnancy; obstruction; small intestine volvulus.

Copyright @ 2020: This is an open-access article distributed under the terms of the Creative Commons Attribution license which permits unrestricted use, distribution, and reproduction in any medium for non-commercial use (NonCommercial, or CC-BY-NC) provided the original author and source are credited.

\section{INTRODUCTION}

A digestive obstruction rarely complicates the course of a pregnancy, but it is frightening for the mother and the unborn child. Its incidence, as reported in the literature, varies between 1 in 66,343 and 1 in 1,500 [1]. Delay in diagnosis is a constant in the literature. It has its origin in the lack of specificity of the clinic and in the frequent wait-and-see attitude of the workers, paralyzed between the consequences of a radiological paraclinical exploration and the operational risk.

It is through a clinical case of small volvulus occlusion in a pregnant woman at 18 weeks, and a review of the literature that we discuss the pathophysiology and the diagnostic and therapeutic problems of this entity.

\section{Case Presentation}

Mrs. H.H aged 38, second primiparous gesture. Admitted to obstetric emergencies for threat of abortion at 18 weeks of gestation with generalized abdominal pain. In these antecedents, the patient presented repetitive genital infections repeatedly treated several times, on the other hand the patient was never operated and her current pregnancy was not followed.

The onset of symptomatology dates back to 3 days with excruciating pain at the peri-umbilical level with vomiting and the notion of stopping of materials and gases followed by a progressive deterioration of the general state then the appearance of pelvic pain. Related to the onset of abortion and febrile syndrome.

The clinical examination on admission found a conscious patient, in hypotension at 09/05, tachycardium at $120 \mathrm{BPM}$, febrile at $39 \mathrm{C}$. the abdominal examination found a slightly distended abdomen with peri-umbilical tenderness and empty rectal bulb at Rectal touch. The obstetrical examination found an open cervix with active endo-uterine bleeding, 1 hour after the patient made a complete expulsion of the product of conception.

The biological assessment showed a leukocytosis at 20,000 elements / $\mathrm{mm} 3$, anemia with $\mathrm{HB}=8.8 \mathrm{~g} / \mathrm{l}$, and a very high CRP at $604 \mathrm{mg} / 1$, uremia at $1 \mathrm{~g} / 1$ with normal serum creatinemia.

The ASP has shown several hail-like hydroaeric levels (Figure 1). The abdominal ultrasound showed distended digestive loops with medium abundance fluid effusion. Radiological exploration was completed by an abdominal scanner (Figure 2) which showed a small mechanical type of occlusion with handles measuring $50 \mathrm{~mm}$ in diameter, with a zone of disparity in caliber and a wall thinned and not enhanced by several handles over time arterial, in favor of a small necrosis, as well as an intraperitoneal effusion of medium abundance. 
Therefore we indicated an emergency laparotomy, after a short preparation in intensive care. The incision is median xyphopubienne after aspiration of about $700 \mathrm{cc}$ of bloody liquid, exploration is volvulus with necrosis of the ileum about a mesenteric flange (Figure 3). We conducted a first peritoneal toilet and resection of necrotic portion (Figure 4) (about $1.2 \mathrm{~m}$ ) to its interesting ileum stoma in the cecum, the ileal stump landfill and finally finished by construction of a terminal ileostomy and drainage of the peritoneal cavity.

The postoperative course was simple, after stay in the intensive care unit for 2 days the patient left the hospital on day 7, and came back 2 months to restore digestive continuity.

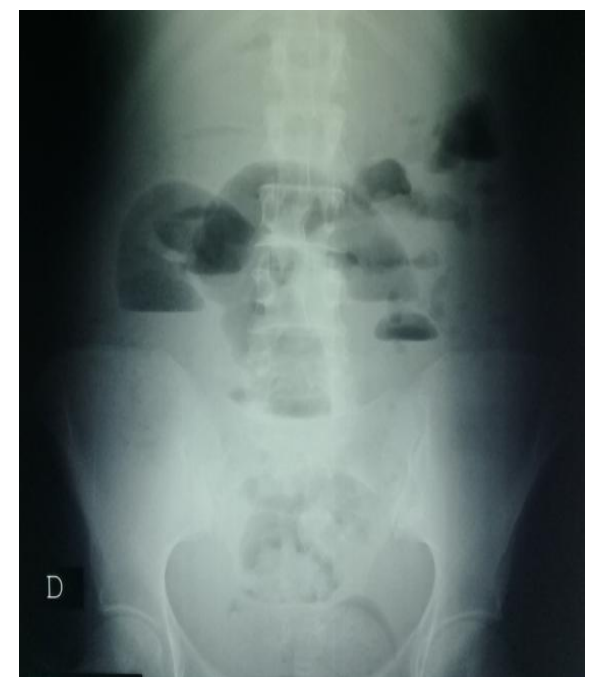

Fig-1: ASP showing hail-like hydro-aeric levels

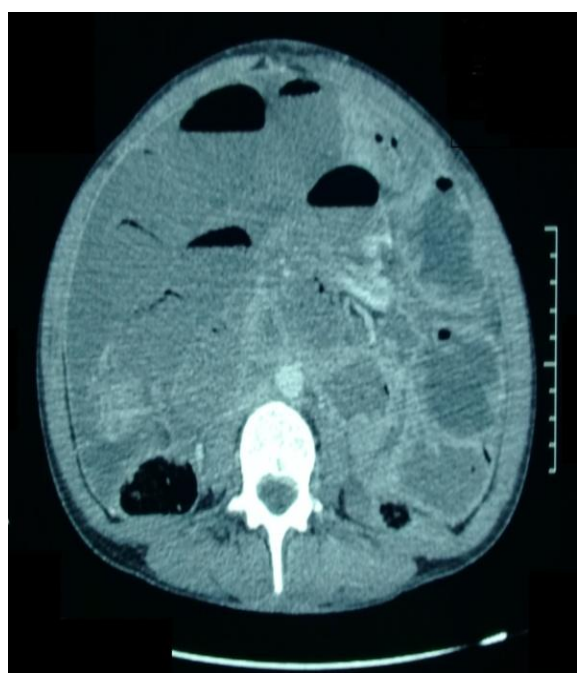

Fig-2: CT aspect of occlusion with grelic necrosis

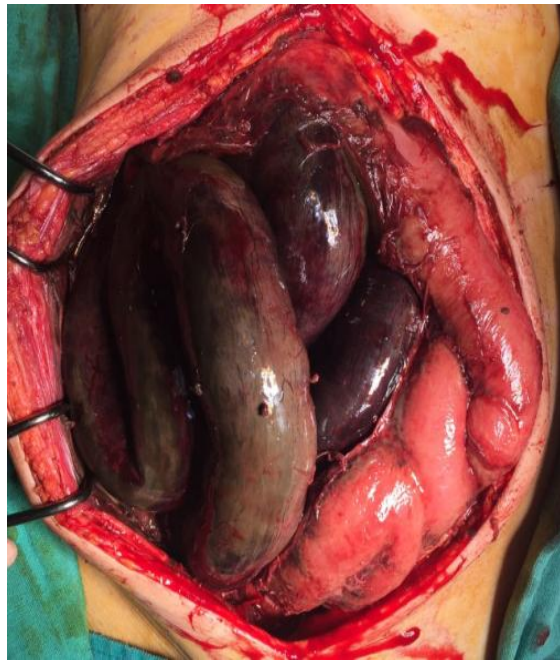

Fig-3: Perioperative aspect of the distended and necrotic small intestine

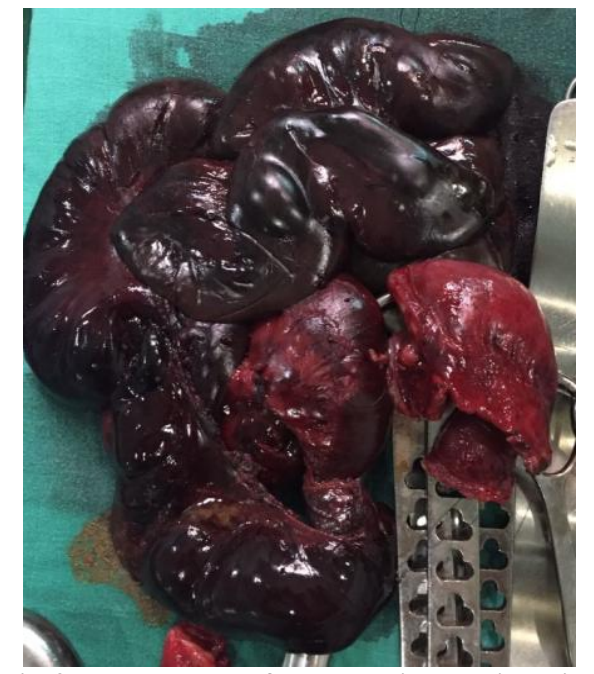

Fig-4: Resected part of the necrotic small intestine

\section{DISCUSSION}

A digestive obstruction rarely complicates the course of a pregnancy. Its incidence varies between $1 /$ 1,500 to $1 / 66,000$ pregnancies. This incidence is increasing; the resurgence of inflammatory pelvic disease (PID) is partly responsible. About 53-59\% of intestinal obstruction due to adhesions or secondary flange surgery or PID episodes [2,3]. The other etiologies are in order of frequency: intestinal volvulus (25\%), acute intestinal intussusception (5\%), and strangulated hernia $(1.4 \%)$, acute appendicitis $(0.5 \%)$ [2]. The distribution according to the trimester of pregnancy is as follows: $6 \%$ in the first quarter, $27 \%$ in the second, the third $44 \%$ and $21 \%$ during the postpartum [4]. Three critical periods were identified during pregnancy: between 16 and 20 weeks during the upward movement of the uterus in the pelvis to the abdomen; between 32 and 36 weeks when the fetal head descends into the pelvis and in the immediate postpartum period, when the sudden reduction in the size of the uterus [3]. 
Physiologically changes that accompany pregnancy promotes advantage of the occlusion, thereby decreasing the intestinal peristalsis and increasing habitual constipation due to the progestin impregnation promoting hypotonia of the intestinal smooth muscle, and changes topographic more flanges of the development of the pregnant uterus [2,5]

The diagnosis of intestinal obstruction during pregnancy is based on the same clinical triad that in the general population: abdominal pain, vomiting, stopping materials and gas. The diagnostic difficulty comes from the fact that nausea and vomiting are frequent pathologies and most often banal during pregnancy: around 16 weeks of gestation, $50 \%$ of patients still complain of nausea and $33 \%$ of vomiting [2]. Bowel obstruction can easily be confused with hyperemesis gravidarum, particularly in the first trimester. Therapeutic responses to the administration of antiemetics are therefore crucial: according to Connoly and Perdue "A lack of response to antiemetics requires further in-depth assessment and must raise the possibility of intestinal obstruction" [2,4].

Abdominal pain and gas shutdown are sufficient warning signs to suggest a diagnosis of bowel obstruction. Abdominal pain is present in 85 to $98 \%$ of cases (5), vomiting in $82 \%$ of cases and stopping of materials in $30 \%$ [2].

All of this accounts for the difficulty of clinical diagnosis (4 cases of misdiagnosis out of 9 patients in the series by Meyerson et al.) And a constant delay in treatment. The average time between admission and surgery is therefore 48 to 84 hours $[6,7]$. Imagery then becomes essential.

Biological analyzes are often inconclusive; Pregnancy is accompanied by physiological hyper leukocytosis which can vary between 9000 to 12000 due to the increase in adrenal cortical activity and therefore would be an unreliable indicator in pregnant women $[2,8,9]$. However, a significant increase in leukocytosis within a few hours should draw attention [4].

Abdominal ultrasound during pregnancy is the first-line diagnostic test for suspected intestinal obstruction $[9,10]$. It allows:

- to exclude an intestinal obstruction with a sensitivity of $89 \%$ and a specificity of $100 \%$, Its safety authorizes repeated examinations which make it possible to follow the evolution of the dilation of the intestinal loops provided that the abundance of gas does not interfere performing the exam.

- To follow the evolution of the dilation of the intestinal loops.

- Detect the presence or abundance of intraperitoneal fluid.
- Appreciate the thickening of the wall indicating venous stasis, ischemia or infarction in the event of volvulus.

The abdomen without preparation loses its specificity as the age of pregnancy. Carried out standing, it frequently shows unspecific hydroaeric levels. Done supine, the topography of the digestive hyper-clarity can orient towards the small intestine or the colon. Finally, 20\% of exams are not contributory: these are beginners $[4,6]$. Given its low intake, it will immediately be replaced by ultrasound. Nuclear magnetic resonance, if the center has it, can be used for differential diagnosis because of its harmlessness to the fetus beyond the first trimester and the information it can provide [11]. While computed tomography is not recommended during pregnancy, except exceptionally when the potential advantage justifies the risk for the fetus and on a case-by-case basis, in close consultation with the obstetrician and the digestive surgeon. In our case the woman has already had an abortion and the indication to have an abdominal scan was easy, which helped to reach the diagnosis of occlusion easily. The majority of observations in the literature describe a medical practice aimed at instituting conservative treatment in the hope of avoiding surgical treatment. This attitude does not seem appropriate. Isolated medical treatment most often ends in failure. Surgical intervention is still performed in $89 \%$ of intestinal obstruction occurring during pregnancy $[2,3,12]$.

The principle of the treatment varies according to the gestational age: Up to 26 weeks: laparotomy with lifting of the occlusion, continuation of the pregnancy until its term if possible; between 26 and 34 weeks: if possible, fetal pulmonary maturation followed by cesarean section with median skin incision supplemented by surgical treatment of the occlusion; between 34 weeks and the term: cesarean section with midline skin incision completed with surgical treatment of the occlusion; in all cases, surgical treatment must be undertaken within 72 hours to avoid major complications of necrosis, perforation and septic shock [4].

\section{CONCLUSION}

Bowel obstruction is a rare complication of pregnancy, Diagnosis is often difficult and late because Digestive disorders are often blamed on pregnancy; however their persistence or their appearance after the 1 st trimester should worry the clinician and encourage him to request the appropriate additional examinations. Management must be multidisciplinary, radiological, obstetric and surgical.

\section{REFERENCES}

1. Twite NC, Jacquet S. Hollemaert. [Intestinal Obstruction in Pregnancy]. Rev Med Brux. 2006. 27(2): 104-9. 
2. Perdue PW, Johnson HW, Staffort PW: Intestinal obstruction complicating pregnancy. Am J Surg. 1992; 164: 384-8

3. Goldthrop WO. Intestinal obstruction during pregnancy and puerperium. Br J Clin Pract. 1966 ; $20: 368-76$

4. Connolly MM, Unti JA, Nora PF. Bowel obstruction in pregnancy. Surg Clin North Am. 1995; 75: 101-3.

5. Beck WW. Intestinal obstruction in pregnancy. Obstet Gynecol. 1974; 43:374-8.

6. Houston J, cited by Hansen F. Intestinal obstruction in the fourth month of pre- gnancy due to adhesions. J Iowa Med Soc. 1941; 31:237.

7. Wax JR, Christie TL. Complete small bowel volvulus complicating the second trimester. Obstet Gynecol. 1993; 82:689- 91.
8. Chang YT, Huang YS, Chan HM, Chan HM, Huang CJ, Hsieh JS, Huang TJ. Intestinal obstruction during pregnancy. Kaohsiung J Med Sci. 2006 Jan; 22(1):20-3.

9. Musoke F, Kawooya MG, Kiguli-Malwadde E. Comparison between sonographic and plain radiography in the diagnosis of small bowel obstruction at Mulago Hospital, Uganda. East Afr Med J. 2003 Oct;80(10):540-5.

10. Bourque, M.R. and J.M. Gibbons, Jr., Intussusception Causing Intestinal Obstruction in Pregnancy. Conn Med, 1979. 43(3): 130-3.

11. Juglard R, Rimbot A, Marty A. Bowel obstruction in pregnancy: value of Single Shot Fast Spin Echo MR sequence (SS-FSE) J Radiol. 2003; 84:1986-8.

12. Meyerson S, Holtz T, Ehrinpreis M, Dhar R. Small bowel obstruction in pregnancy. Am J Gastroenterol. 1995; 90:299-302. 